\title{
INFINITE PRODUCTS OF HOLOMORPHIC MAPPINGS
}

\author{
MONIKA BUDZYŃSKA AND SIMEON REICH
}

Received 14 September 2004

Let $X$ be a complex Banach space, $\mathcal{N}$ a norming set for $X$, and $D \subset X$ a bounded, closed, and convex domain such that its norm closure $\bar{D}$ is compact in $\sigma(X, \mathcal{N})$. Let $\varnothing \neq C \subset D$ lie strictly inside $D$. We study convergence properties of infinite products of those selfmappings of $C$ which can be extended to holomorphic self-mappings of $D$. Endowing the space of sequences of such mappings with an appropriate metric, we show that the subset consisting of all the sequences with divergent infinite products is $\sigma$-porous.

\section{Introduction}

Let $K$ be a nonempty, bounded, closed, and convex subset of a Banach space. The convergence of infinite products of self-mappings of such sets is of interest in many areas of mathematics and its applications. See, for instance, [19] and references therein. In a recent paper [20], it is proved that the subsets consisting of all those sequences of nonexpansive self-mappings of $K$ with divergent infinite products are not only of the first Baire category, but also $\sigma$-porous in several spaces, endowed with appropriate metrics, of sequences of such mappings. In the present paper, we establish analogous results for holomorphic mappings. After discussing some basic facts regarding holomorphic mappings and the Kobayashi distance in the next section, we study weak ergodicity in Section 3. The convergence of infinite products to a (unique) common fixed point is considered in Section 4. In the last section of our paper, we study the convergence of infinite products to a retraction.

\section{Preliminaries}

In this section, we recall several basic facts concerning the Kobayashi distance and holomorphic mappings. These facts will be used throughout our paper.

In the sequel, all Banach spaces $X$ will be complex and $D$ will always denote a bounded, convex domain in $X$. Let $k_{D}$ be the Kobayashi distance in $D[15]$ (see also $[10,11,12,13$, 17]).

We first quote the following very useful lemma regarding convex combinations of points. 
Lemma 2.1 [18] (see also [17]). Let D be a bounded, convex domain in a Banach space $(X,\|\cdot\|)$.

(i) If $x, y, w, z \in D$ and $s \in[0,1]$, then

$$
k_{D}(s x+(1-s) y, s w+(1-s) z) \leq \max \left[k_{D}(x, w), k_{D}(y, z)\right] .
$$

(ii) If $x, y \in D$ and $s, t \in[0,1]$, then

$$
k_{D}(s x+(1-s) y, t x+(1-t) y) \leq k_{D}(x, y) .
$$

There are also simple connections between $k_{D}$ and the norm $\|\cdot\|$ of $X$.

Theorem $2.2[12,17]$. If $D$ is a bounded, convex domain in a Banach space $(X,\|\cdot\|)$, then

$$
\arg \tanh \left(\frac{\|x-y\|}{\operatorname{diam}_{\|\cdot\|} D}\right) \leq k_{D}(x, y)
$$

for all $x, y \in D$ and

$$
k_{D}(x, y) \leq \arg \tanh \left(\frac{\|x-y\|}{\operatorname{dist}_{\|\cdot\|}(x, \partial D)}\right)
$$

whenever $\|x-y\|<\operatorname{dist}_{\|\cdot\|}(x, \partial D)$.

This theorem shows that the Kobayashi distance $k_{D}$ is locally equivalent to the norm $\|\cdot\|$.

Next, we observe that in analogy with the norm, the Kobayashi distance is lower semicontinuous with respect to a suitably chosen topology. Let $\mathcal{N}$ be a nonempty subset of the dual $X^{*}$ of $X$. If there exist positive constants $r$ and $R$ such that

$$
\sup \{|l(x)|: l \in \mathcal{N},\|l\| \leq R\} \geq r\|x\|
$$

for each $x \in X$, then we say that $\mathcal{N}$ is a norming set for $X$ [8]. It is obvious that a norming set generates a Hausdorff linear topology $\sigma(X, \mathcal{N})$ on $X$ which is weaker than the weak topology $\sigma\left(X, X^{*}\right)$.

Theorem 2.3 [14] (see also $[2,16,17]$ ). Let $X$ be a Banach space, $\mathcal{N}$ a norming set for $X$, and $D \subset X$ a bounded, convex domain such that its norm closure $\bar{D}$ is compact in the $\sigma(X, \mathcal{N})$ topology. If $\left\{x_{\beta}\right\}_{\beta \in J}$ and $\left\{y_{\beta}\right\}_{\beta \in J}$ are nets in $D$ which are convergent in $\sigma(X, \mathcal{N})$ to $x$ and $y$, respectively, and $x, y \in D$, then

$$
k_{D}(x, y) \leq \liminf _{\beta} k_{D}\left(x_{\beta}, y_{\beta}\right) .
$$

If the nets $\left\{x_{\beta}\right\}_{\beta \in J}$ and $\left\{y_{\beta}\right\}_{\beta \in J}$ are replaced by the sequences $\left\{x_{k}\right\}_{k \in \mathbb{N}}$ and $\left\{y_{k}\right\}_{k \in \mathbb{N}}$, then the compactness of $\bar{D}$ in $\sigma(X, \mathcal{N})$ can be replaced by its sequential compactness in $\sigma(X, \mathcal{N})$.

Recall that a subset $C$ of $D$ is said to lie strictly inside $D$ if $\operatorname{dist}_{\|\cdot\|}(C, \partial D)>0$. Thus any closed subset $C$ lying strictly inside a bounded, convex domain $D$ is complete with respect to $k_{D}$. 
Theorem 2.4 [12]. Let $D$ be a bounded, convex domain in a Banach space $(X,\|\cdot\|)$. A subset $C$ of $D$ is $k_{D}$-bounded if and only if C lies strictly inside $D$.

Now, we present a few results concerning holomorphic mappings. We begin with the definition of a holomorphic mapping. Let $X_{1}$ and $X_{2}$ be two complex normed linear spaces and let $D_{1}$ be a domain in $X_{1}$. A mapping $f: D_{1} \rightarrow X_{2}$ is said to be holomorphic in $D_{1}$ if it is Fréchet differentiable at each point of $D_{1}$. An equivalent definition is given in the following theorem.

Theorem 2.5 [8] (see also [3]). Let $\left(X_{1},\|\cdot\|_{1}\right)$ and $\left(X_{2},\|\cdot\|_{2}\right)$ be Banach spaces, $D$ a domain in $X_{1}$, and let $\mathcal{N}$ be a norming set for $\left(X_{2},\|\cdot\|_{2}\right)$. For $a \in D$ and $x \in X_{1} \backslash\{0\}$, let $D(a, x)$ denote the set

$$
D(a, x)=\{z \in \mathbb{C}: a+z x \in D\} .
$$

Then the mapping $f: D \rightarrow X_{2}$ is holomorphic in $D$ if and only if $f$ is locally bounded on $D$ and for each $a \in D, x \in X_{1} \backslash\{0\}$, and $l \in \mathcal{N}$, the function

$$
l \circ f_{\mid D(a, x)}: D(a, x) \longrightarrow \mathbb{C}
$$

is holomorphic in $D(a, x)$ in the classical one-variable sense.

Directly from Theorem 2.5, we get the following simple lemma regarding nets of holomorphic mappings. We note here that $\bar{C}$ always stands for the norm closure of each subset $C$ of a Banach space.

Lemma 2.6 [14] (see also [16]). Let $D_{1}$ and $D_{2}$ be bounded, convex domains in the $B a$ nach spaces $\left(X_{1},\|\cdot\|_{1}\right)$ and $\left(X_{2},\|\cdot\|_{2}\right)$, respectively, and $\mathcal{N}$ a norming set for $\left(X_{2},\|\cdot\|_{2}\right)$. If $\left\{f_{\lambda}\right\}_{\lambda \in J}$ is a net of holomorphic mappings $f_{\lambda}: D_{1} \rightarrow D_{2}$ which is pointwise convergent in the topology $\sigma\left(X_{2}, \mathcal{N}\right)$ to a mapping $f: D_{1} \rightarrow \overline{D_{2}}$ and there exists a point $z_{0} \in D_{1}$ such that $w_{0}=f\left(z_{0}\right) \in D_{2}$, then $f$ maps $D_{1}$ holomorphically into $D_{2}$.

Let $\left(M_{1}, d_{1}\right)$ and $\left(M_{2}, d_{2}\right)$ be two metric spaces. We say that a mapping $f: M_{1} \rightarrow M_{2}$ is nonexpansive if

$$
d_{2}(f(x), f(y)) \leq d_{1}(x, y)
$$

for all $x, y \in M_{1}$.

An immediate consequence of the definition of the Kobayashi distance is the following property of holomorphic mappings. If $D_{1}$ and $D_{2}$ are bounded domains in the Banach spaces $\left(X_{1},\|\cdot\|_{1}\right)$ and $\left(X_{2},\|\cdot\|_{2}\right)$, respectively, and $k_{D_{1}}$ and $k_{D_{2}}$ are the Kobayashi distances on $D_{1}$ and $D_{2}$, respectively, then each holomorphic $f: D_{1} \rightarrow D_{2}$ is nonexpansive, that is,

$$
k_{D_{1}}(f(x), f(y)) \leq k_{D_{2}}(x, y)
$$

for all $x, y \in D_{1}$ [15].

We also recall the Earle-Hamilton theorem. 
Theorem 2.7 [9]. Let $D$ be a bounded, convex domain in a Banach space $(X,\|\cdot\|)$ and let $\widetilde{R}=\operatorname{diam}_{\|\cdot\|} D$. Let a holomorphic $f: D \rightarrow D$ map D strictly inside itself. If $\epsilon>0$ is such that $\operatorname{dist}_{\|\cdot\|}(f(D), \partial D) \geq \epsilon$ and $t=\epsilon / 2 \widetilde{R}$, then for $0<s=1 /(1+t)<1$,

$$
k_{D}(f(x), f(y)) \leq s k_{D}(x, y)
$$

for all $x, y \in D$, and therefore $f$ has a unique fixed point. Moreover, for any $x$ in $D$, the sequence of iterates $\left\{f^{k}(x)\right\}$ converges to this fixed point.

Now we introduce basic notions and notations concerning those spaces which we will investigate in subsequent sections.

Throughout this paper, we let $X$ be a complex Banach space, $\mathcal{N}$ a norming set for $X$, and $D \subset X$ a bounded, closed, and convex domain such that its norm closure $\bar{D}$ is compact in $\sigma(X, \mathcal{N})$. Let $C$ be a $k_{D}$-bounded subset of $D$ and let the set $\boldsymbol{U}_{H}$ consist of all those self-mappings of $C$ which can be extended to holomorphic self-mappings of $D$. We endow $u_{H}$ with the metric $\rho u_{H}$ defined by

$$
\rho{\cup_{H}}_{H}(f, g)=\sup _{x \in C} k_{D}(f(x), g(x))
$$

for $f, g \in U_{H}$. It is not difficult to see that, by Lemma 2.6, the metric space $\left(u_{H}, \rho u_{H}\right)$ is complete.

Denote by $\mathscr{A}_{H}$ the set of all sequences $\left\{f_{t}\right\}_{t=1}^{\infty}$, where each $f_{t} \in \mathcal{U}_{H}$. We equip the space $\mathscr{A}_{H}$ with the metric $d_{\mathscr{A}_{H}}$ defined by

$$
d_{\mathscr{A}_{H}}\left(\left\{f_{t}\right\}_{t=1}^{\infty},\left\{g_{t}\right\}_{t=1}^{\infty}\right)=\sup _{t \geq 1} \rho{\Upsilon_{H}}_{H}\left(f_{t}, g_{t}\right)
$$

where $\left\{f_{t}\right\}_{t=1}^{\infty},\left\{g_{t}\right\}_{t=1}^{\infty} \in \mathscr{A}_{H}$. Clearly, the metric space $\left(\mathscr{A}_{H}, d_{\mathscr{A}_{H}}\right)$ is also complete.

Finally, we recall the concept of porosity. We will use the rather strong notion which appears in $[4,5,6,7]$. In the literature, one can also find other notions of porosity $[1,21$, $22,23]$.

Definition 2.8. Let $(Y, d)$ be a complete metric space. Denote by $B(x, R)$ the closed ball centered at $x \in Y$ and of radius $R>0$. A subset $E \subset Y$ is called porous in $(Y, d)$ if there exist $\alpha \in(0,1)$ and $R_{0}>0$ such that for each $R \in\left(0, R_{0}\right)$ and each $y \in Y$, there is a point $z \in Y$ for which

$$
B(z, \alpha R) \subset B(y, R) \backslash E .
$$

A subset of the space $Y$ is called $\sigma$-porous in $(Y, d)$ if it is a countable union of porous subsets in $(Y, d)$.

To end this section, we introduce the following notations which will be used throughout this paper.

Let $D \subset X$ be a bounded, closed, and convex domain and let $C$ be a $k_{D}$-bounded subset of $D$. The positive numbers $R_{1}, R_{2}$ are such that for each $\tilde{x}_{0} \in C$, the closed ball 
$B_{\|\cdot\|}\left(\tilde{x}_{0}, R_{1}\right)$ in $(X,\|\cdot\|)$ lies in $D$ and $\operatorname{diam}_{\|\cdot\|} D<R_{2}$. Next, $0<L_{1}<L_{2}$ satisfy

$$
L_{1} k_{D}(x, y) \leq\|x-y\| \leq L_{2} k_{D}(x, y)
$$

for all $x, y \in C$. It is obvious that $0<R_{1} / R_{2}<1$ and $K=L_{2} / L_{1}>1$.

If $\varnothing \neq C \subset D$, then $d(C)$ denotes the diameter of $C$ in $\left(D, k_{D}\right)$, that is,

$$
d(C)=\sup _{x, y \in C} k_{D}(x, y)
$$

\section{Weak ergodicity}

This section is devoted to weak ergodicity in the sense of population biology (see [19] and the references therein). Our result is analogous to [20, Theorem 1.1].

A sequence $\left\{f_{t}\right\}_{t=1}^{\infty} \in \mathscr{A}_{H}$ is called regular if for any $\epsilon>0$, there exists a number $N \in$ $\mathbb{N}$ such that for each $x, y \in C$, each integer $T \geq N$, and each mapping $\pi:\{1, \ldots, T\} \rightarrow$ $\{1,2, \ldots\}$, we have

$$
k_{D}\left(\left(f_{\pi(T)} \circ \cdots \circ f_{\pi(1)}\right)(x),\left(f_{\pi(T)} \circ \cdots \circ f_{\pi(1)}\right)(y)\right) \leq \epsilon .
$$

A mapping $f \in U_{H}$ is called regular if the sequence $\hat{f}=\left\{f_{t}\right\}_{t=1}^{\infty}$, where $f_{t}=f(t \geq 1)$, is regular. It is easy to verify that if $f \in \mathcal{U}_{H}$ is regular, then there exists a unique $x_{f} \in C$ such that $f\left(x_{f}\right)=x_{f}$ and $f^{n}(x) \rightarrow x_{f}$ as $n \rightarrow \infty$, uniformly on $C$.

Denote by $\mathscr{F}_{F}$ the set of all regular elements of $\mathscr{A}_{H}$ and by $\mathscr{F}_{(0)}$ the set of all regular elements of $u_{H}$.

For each $n \in \mathbb{N}$, we denote by $\mathscr{F}_{n}$ the set of all sequences $\left\{f_{t}\right\}_{t=1}^{\infty} \in \mathscr{A}_{H}$ which have the following property.

There exists an integer $N \in \mathbb{N}$ such that for each $x, y \in C$, each integer $T \geq N$, and each mapping $\pi:\{1, \ldots, T\} \rightarrow\{1,2, \ldots\}$,

$$
k_{D}\left(\left(f_{\pi(T)} \circ \cdots \circ f_{\pi(1)}\right)(x),\left(f_{\pi(T)} \circ \cdots \circ f_{\pi(1)}\right)(y)\right) \leq \frac{1}{n}
$$

It is not difficult to see that $\mathscr{F}=\bigcap_{n=1}^{\infty} \mathscr{F}_{n}$.

Similarly, $\mathscr{F}_{n}^{(0)}$ is the set of all $f \in \mathcal{U}_{H}$ such that $\hat{f} \in \mathscr{F}_{n}$. Clearly, $\mathscr{F}^{(0)}=\bigcap_{n=1}^{\infty} \mathscr{F}_{n}^{(0)}$.

Theorem 3.1. Under the assumptions given in the definitions of $\mathscr{A}_{H}, \mathscr{U}_{H}, \mathscr{F}_{\text {, and }} \mathscr{F}^{(0)}$,

(i) the set $\mathscr{A}_{H} \backslash \mathscr{F}$ is $\sigma$-porous in $\mathscr{A}_{H}$,

(ii) the set $\boldsymbol{U}_{H} \backslash \mathscr{F}^{(0)}$ is $\sigma$-porous in $\boldsymbol{U}_{H}$.

Proof. We will show that $\mathscr{A}_{H} \backslash \mathscr{F}_{n}$ is porous in $\mathscr{A}_{H}$ and that $\boldsymbol{U}_{H} \backslash \mathscr{F}_{n}^{(0)}$ is porous in $\boldsymbol{U}_{H}$ for each $n \in \mathbb{N}$. To this end, fix $n \in \mathbb{N}$ and choose $\alpha \in(0,1)$ such that

$$
\alpha<\frac{1-\alpha}{8 K n(d(C)+1)} \cdot \frac{R_{1}}{3 R_{2}}
$$


where $R_{1}, R_{2}, K$, and $d(C)$ are defined at the end of the previous section. Assume that $\left\{f_{t}\right\}_{t=1}^{\infty} \in \mathscr{A}_{H}$ and $R \in(0,1]$. Set

$$
\gamma=\frac{(1-\alpha) R}{2 K(d(C)+1)} \cdot \frac{R_{1}}{3 R_{2}}=s \frac{R_{1}}{3 R_{2}},
$$

where $0<s<1$, and choose $0<N \in \mathbb{N}$ such that

$$
d(C)+1<2 N \alpha R
$$

Observe that

$$
4 \alpha n R<\gamma
$$

Fix $x^{\prime} \in C$. For each $t \in \mathbb{N}$, define

$$
f_{\gamma t}(x)=(1-s) f_{t}(x)+s x^{\prime},
$$

where $x \in D$. Note that if $f_{t}=f(t \geq 1)$ with $f \in \mathcal{U}_{H}$, then $f_{\gamma t}=f_{\gamma}(t \geq 1)$, where

$$
f_{\gamma}(x)=(1-s) f(x)+s x^{\prime}
$$

for $x \in D$. Hence, we get

$$
\begin{aligned}
k_{D}\left(f_{\gamma t}(x), f_{t}(x)\right) & \leq \frac{1}{L_{1}} s\left\|f_{t}(x)-x^{\prime}\right\| \leq \frac{L_{2}}{L_{1}} s k_{D}\left(f_{t}(x), x^{\prime}\right) \leq K \gamma \frac{3 R_{2}}{R_{1}} d(C) \\
& =K \cdot \frac{(1-\alpha) R}{2 K(d(C)+1)} \cdot \frac{R_{1}}{3 R_{2}} \cdot \frac{3 R_{2}}{R_{1}} \cdot d(C)<\frac{(1-\alpha) R}{2}
\end{aligned}
$$

for each $x \in C$, and

$$
\begin{aligned}
k_{D}\left(f_{\gamma t}(x), f_{\gamma t}(y)\right) & \leq \frac{1}{1+s R_{1} / 2 R_{2}} k_{D}(x, y)=\left(1-\frac{s R_{1}}{2 R_{2}+s R_{1}}\right) k_{D}(x, y) \\
& \leq\left(1-s \frac{R_{1}}{3 R_{2}}\right) k_{D}(x, y)=(1-\gamma) k_{D}(x, y)
\end{aligned}
$$

for every $x, y \in D$. Now, assume that $\left\{g_{t}\right\}_{t=1}^{\infty} \in \mathscr{A}_{H}$ and that

$$
d_{\mathbb{A}_{H}}\left(\left\{f_{\gamma t}\right\}_{t=1}^{\infty},\left\{g_{t}\right\}_{t=1}^{\infty}\right) \leq \alpha R
$$

Then we see that

$$
\begin{aligned}
d_{\mathscr{A}_{H}}\left(\left\{f_{t}\right\}_{t=1}^{\infty},\left\{g_{t}\right\}_{t=1}^{\infty}\right) & \leq d_{\mathscr{A}_{H}}\left(\left\{f_{\gamma t}\right\}_{t=1}^{\infty},\left\{g_{t}\right\}_{t=1}^{\infty}\right)+d_{\mathscr{A}_{H}}\left(\left\{f_{\gamma t}\right\}_{t=1}^{\infty},\left\{f_{t}\right\}_{t=1}^{\infty}\right) \\
& <\alpha R+\frac{(1-\alpha) R}{2}=\frac{(1+\alpha) R}{2}<R .
\end{aligned}
$$

To prove that for each $x, y \in C$, each integer $T \geq N$, and each mapping $\pi:\{1, \ldots, T\} \rightarrow$ $\{1,2, \ldots\}$, we have

$$
k_{D}\left(\left(g_{\pi(T)} \circ \cdots \circ g_{\pi(1)}\right)(x),\left(g_{\pi(T)} \circ \cdots \circ g_{\pi(1)}\right)(y)\right) \leq \frac{1}{n},
$$


it is sufficient to verify that for each $x, y \in C$, each integer $T \geq N$, and each mapping $\pi:\{1, \ldots, N\} \rightarrow\{1,2, \ldots\}$, there is an integer $m \in\{1, \ldots, N\}$ such that

$$
k_{D}\left(\left(g_{\pi(m)} \circ \cdots \circ g_{\pi(1)}\right)(x),\left(g_{\pi(m)} \circ \cdots \circ g_{\pi(1)}\right)(y)\right) \leq \frac{1}{n} .
$$

To this end, assume that $x, y \in C$ and $\pi:\{1, \ldots, N\} \rightarrow\{1,2, \ldots\}$. Assume, contrary to our claim, that for each integer $j \in\{1, \ldots, N\}$,

$$
\begin{gathered}
k_{D}\left(\left(g_{\pi(j)} \circ \cdots \circ g_{\pi(1)}\right)(x),\left(g_{\pi(j)} \circ \cdots \circ g_{\pi(1)}\right)(y)\right)>\frac{1}{n}, \\
k_{D}(x, y)>\frac{1}{n} .
\end{gathered}
$$

Set

$$
\begin{array}{ll}
x_{0}=x, & x_{j+1}=g_{\pi(j+1)} x_{j}, \\
y_{0}=y, & y_{j+1}=g_{\pi(j+1)} y_{j}
\end{array}
$$

for each $j \in\{0, \ldots, N-1\}$.

Now fix $j \in\{0, \ldots, N-1\}$. Then

$$
k_{D}\left(x_{j}, y_{j}\right)>\frac{1}{n}
$$

Moreover,

$$
k_{D}\left(f_{\gamma \pi(j+1)}\left(x_{j}\right), f_{\gamma \pi(j+1)}\left(y_{j}\right)\right) \leq(1-\gamma) k_{D}\left(x_{j}, y_{j}\right) .
$$

It follows from the above inequalities that

$$
\begin{aligned}
k_{D}\left(x_{j+1}, y_{j+1}\right)= & k_{D}\left(g_{\pi(j+1)}\left(x_{j}\right), g_{\pi(j+1)}\left(y_{j}\right)\right) \\
\leq & k_{D}\left(g_{\pi(j+1)}\left(x_{j}\right), f_{\gamma \pi(j+1)}\left(x_{j}\right)\right)+k_{D}\left(f_{\gamma \pi(j+1)}\left(x_{j}\right), f_{\gamma \pi(j+1)}\left(y_{j}\right)\right) \\
& +k_{D}\left(f_{\gamma \pi(j+1)}\left(y_{j}\right), g_{\pi(j+1)}\left(y_{j}\right)\right) \\
\leq & (1-\gamma) k_{D}\left(x_{j}, y_{j}\right)+2 \alpha R=k_{D}\left(x_{j}, y_{j}\right)-\gamma k_{D}\left(x_{j}, y_{j}\right)+2 \alpha R \\
< & k_{D}\left(x_{j}, y_{j}\right)-\frac{\gamma}{n}+2 \alpha R<k_{D}\left(x_{j}, y_{j}\right)-\frac{4 \alpha n R}{n}+2 \alpha R \\
= & k_{D}\left(x_{j}, y_{j}\right)-2 \alpha R .
\end{aligned}
$$

Therefore, we obtain

$$
k_{D}\left(x_{N}, y_{N}\right) \leq k_{D}\left(x_{0}, y_{0}\right)-2 N \alpha R \leq d(C)-2 N \alpha R<0 \text {. }
$$

This contradiction yields the existence of an integer $m \in\{1, \ldots, N\}$ for which the inequality

$$
k_{D}\left(\left(g_{\pi(m)} \circ \cdots \circ g_{\pi(1)}\right)(x),\left(g_{\pi(m)} \circ \cdots \circ g_{\pi(1)}\right)(y)\right) \leq \frac{1}{n}
$$


is valid. Hence $\left\{g_{t}\right\}_{t=1}^{\infty} \in \mathscr{F}_{n}$. Thus we have shown that

$$
\begin{aligned}
& \left\{\left\{g_{t}\right\}_{t=1}^{\infty} \in \mathscr{A}_{H}: d_{\mathscr{A}_{H}}\left(\left\{f_{\gamma t}\right\}_{t=1}^{\infty},\left\{g_{t}\right\}_{t=1}^{\infty}\right) \leq \alpha R\right\} \\
& \subset\left\{\left\{\tilde{f}_{t}\right\}_{t=1}^{\infty} \in \mathscr{A}_{H}: d_{\mathscr{A}_{H}}\left(\left\{f_{t}\right\}_{t=1}^{\infty},\left\{\tilde{f}_{t}\right\}_{t=1}^{\infty}\right)<R\right\} \cap \mathscr{F}_{n} .
\end{aligned}
$$

If $f \in \mathcal{U}_{H}$ and $f_{t}=f$ for all $t \geq 1$, then $f_{\gamma t}=f_{\gamma}(t \geq 1)$ and

$$
\left\{g \in U_{H}: \rho{U_{H}}_{H}\left(f_{\gamma}, g\right) \leq \alpha r\right\} \subset\left\{\tilde{f} \in U_{H}: \rho u_{H}(f, \tilde{f})<r\right\} \cap \mathscr{F}_{n}^{(0)} .
$$

Consequently, the set $\mathscr{A}_{H} \backslash \mathscr{F}_{n}$ is porous in $\mathscr{A}_{H}$ and the set $\varkappa_{H} \backslash \mathscr{F}_{n}^{(0)}$ is porous in $\boldsymbol{U}_{H}$ for each $n \in \mathbb{N}$. This completes the proof.

\section{Convergence to common fixed points}

In this section, we will study the convergence of unrestricted infinite products to a common fixed point.

First we introduce the following notations. Let $\mathscr{A}_{H}^{*}$ denote the set of all sequences $\mathbf{f}=\left\{f_{t}\right\}_{i=1}^{\infty} \in \mathscr{A}_{H}$ for which there exists $x_{\mathbf{f}} \in C$ such that

$$
f_{t}\left(x_{\mathbf{f}}\right)=x_{\mathbf{f}}
$$

for all $t \geq 1$. The closure of $\mathscr{A}_{H}^{*}$ in the metric space $\left(\mathscr{A}_{H}, d_{\mathscr{A}_{H}}\right)$ will be denoted by $\overline{\mathscr{A}_{H}^{*}}$.

THEOREM 4.1. Let $\mathscr{F}$ be the set of all $\mathbf{f}=\left\{f_{t}\right\}_{i=1}^{\infty} \in \overline{\mathbb{A}_{H}^{*}}$ which satisfy the following conditions:

(i) there exists $x_{*} \in C$ such that $f_{t}\left(x_{*}\right)=x_{*}$ for all $t \geq 1$;

(ii) for each $\epsilon>0$, there exists an $N \in \mathbb{N}$ such that

$$
k_{D}\left(\left(f_{\pi(n)} \circ \cdots \circ f_{\pi(1)}\right)(x), x_{*}\right) \leq \epsilon
$$

for each integer $n \geq N$, each mapping $\pi:\{1, \ldots, n\} \rightarrow\{1,2, \ldots\}$, and each $x \in C$.

Then the set $\overline{\mathscr{A}_{H}^{*}} \backslash \mathscr{F}$ is $\sigma$-porous in $\overline{\mathscr{A}_{H}^{*}}$.

Proof. For each $n \in \mathbb{N}$, let $\mathscr{F}_{n}$ be the set of all sequences $\left\{f_{t}\right\}_{i=1}^{\infty} \in \overline{\mathscr{A}_{H}^{*}}$ for which there exist $x^{(n)} \in C$ and an $N \in \mathbb{N}$ such that

$$
k_{D}\left(\left(f_{\pi(T)} \circ \cdots \circ f_{\pi(1)}\right)(x), x^{(n)}\right) \leq \frac{1}{n}
$$

for each integer $T \geq N$, each mapping $\pi:\{1, \ldots, T\} \rightarrow\{1,2, \ldots\}$, and each point $x \in C$. It is obvious that $\mathscr{F}_{F}=\bigcap_{n=1}^{\infty} \mathscr{F}_{n}$. Now, fix $n \in \mathbb{N}$. We will show that the set $\overline{\mathscr{A}_{H}^{*}} \backslash \mathscr{F}_{n}$ is porous in $\overline{\mathscr{A}}_{H}^{*}$. To see this, let $\alpha \in(0,1)$ be such that

$$
\alpha<\frac{1}{8 K n(d(C)+1)} \cdot \frac{R_{1}}{3 R_{2}}
$$


where $R_{1}, R_{2}, K$, and $d(C)$ are defined in Section 2. Clearly, $0<\alpha<1 / 2$. Assume that $\tilde{\mathbf{f}}=\left\{\tilde{f}_{t}\right\}_{i=1}^{\infty} \in \overline{\mathcal{A}_{H}^{*}}$ and $R \in(0,1]$. Then there exists $\mathbf{f}=\left\{f_{t}\right\}_{i=1}^{\infty} \in \mathscr{A}_{H}^{*}$ such that

$$
d_{\mathscr{A}_{H}}\left(\left\{\tilde{f}_{t}\right\}_{i=1}^{\infty},\left\{f_{t}\right\}_{i=1}^{\infty}\right) \leq \frac{R}{4}
$$

Let $x_{\mathrm{f}} \in C$ satisfy

$$
f_{t}\left(x_{\mathbf{f}}\right)=x_{\mathbf{f}}
$$

for all $t \geq 1$. Set

$$
\gamma=\frac{R}{4 K(d(C)+1)} \cdot \frac{R_{1}}{3 R_{2}}=s \frac{R_{1}}{3 R_{2}} .
$$

It is obvious that $0<\gamma<1$ and $0<s<1$. Next, choose $2<N \in \mathbb{N}$ such that

$$
(1-\gamma)^{N}(d(C)+1)<\frac{1}{2 n} .
$$

Finally, for each $t \in \mathbb{N}$, define

$$
f_{\gamma t}(x)=(1-s) f_{t}(x)+s x_{\mathbf{f}}
$$

for $x \in C$. It is obvious that $\left\{f_{\gamma t}\right\}_{t=1}^{\infty} \in \mathscr{A}_{H}^{*}$. Moreover, we have

$$
\begin{aligned}
k_{D}\left(f_{\gamma t}(x), f_{\gamma t}(y)\right) & \leq \frac{1}{1+s R_{1} / 2 R_{2}} k_{D}(x, y)=\left(1-\frac{s R_{1}}{s R_{1}+2 R_{2}}\right) k_{D}(x, y) \\
& \leq\left(1-s \frac{R_{1}}{3 R_{2}}\right) k_{D}(x, y)=(1-\gamma) k_{D}(x, y)
\end{aligned}
$$

for every $x, y \in D$. Next, we obtain

$$
\begin{aligned}
k_{D}\left(f_{\gamma t}(x), f_{t}(x)\right) & \leq \frac{1}{L_{1}} s\left\|f_{t}(x)-x_{\mathbf{f}}\right\| \leq \frac{L_{2}}{L_{1}} s k_{D}\left(f_{t}(x), x_{\mathbf{f}}\right) \\
& \leq K \frac{3 R_{2}}{R_{1}} \gamma d(C)=K \cdot \frac{3 R_{2}}{R_{1}} \cdot \frac{R}{4 K(d(C)+1)} \cdot \frac{R_{1}}{3 R_{2}} \cdot d(C)<\frac{R}{4}
\end{aligned}
$$

for each $x \in C$. Assume now that $\left\{g_{t}\right\}_{i=1}^{\infty} \in \mathscr{A}_{H}$ and

$$
d_{\mathscr{A}_{H}}\left(\left\{f_{\gamma t}\right\}_{i=1}^{\infty},\left\{g_{t}\right\}_{i=1}^{\infty}\right) \leq \alpha R .
$$

Then we get

$$
\begin{aligned}
d_{\mathscr{A}_{H}}\left(\left\{\tilde{f}_{t}\right\}_{i=1}^{\infty},\left\{g_{t}\right\}_{i=1}^{\infty}\right) \leq & d_{\mathscr{A}_{H}}\left(\left\{\tilde{f}_{t}\right\}_{i=1}^{\infty},\left\{f_{t}\right\}_{i=1}^{\infty}\right)+d_{\mathscr{A}_{H}}\left(\left\{f_{t}\right\}_{i=1}^{\infty},\left\{f_{\gamma t}\right\}_{i=1}^{\infty}\right) \\
& +d_{\mathscr{A}_{H}}\left(\left\{f_{\gamma t}\right\}_{i=1}^{\infty},\left\{g_{t}\right\}_{i=1}^{\infty}\right) \\
& <\frac{R}{4}+\frac{R}{4}+\alpha R<R .
\end{aligned}
$$


We will show that the following property holds.

(P1) For each $x \in C$, each integer $T \geq N$, and each mapping $\pi:\{1, \ldots, T\} \rightarrow\{1,2, \ldots\}$,

$$
k_{D}\left(\left(g_{\pi(T)} \circ \cdots \circ g_{\pi(1)}\right)(x), x_{\mathbf{f}}\right) \leq \frac{1}{n} .
$$

So, let $y \in C$ and $t \in \mathbb{N}$. We have

$$
k_{D}\left(f_{\gamma t}(y), x_{\mathbf{f}}\right)=k_{D}\left(f_{\gamma t}(y), f_{\gamma t}\left(x_{\mathbf{f}}\right)\right) \leq(1-\gamma) k_{D}\left(y, x_{\mathbf{f}}\right),
$$

which implies that

$$
k_{D}\left(g_{t}(y), x_{\mathbf{f}}\right) \leq k_{D}\left(g_{t}(y), f_{\gamma t}(y)\right)+k_{D}\left(f_{\gamma t}(y), x_{\mathbf{f}}\right) \leq \alpha R+(1-\gamma) k_{D}\left(y, x_{\mathbf{f}}\right)
$$

for each $t \in \mathbb{N}$ and each $y \in C$. Assume that $x \in C, T \geq N$, and $\pi:\{1, \ldots, T\} \rightarrow\{1,2, \ldots\}$. Set

$$
\begin{gathered}
x_{0}=x \\
x_{i+1}=g_{\pi(i+1)}\left(x_{i}\right) \quad(i \geq 0) .
\end{gathered}
$$

Then, for any integer $i \in \mathbb{N}_{0}$, we get

$$
k_{D}\left(x_{i+1}, x_{\mathbf{f}}\right)=k_{D}\left(g_{\pi(i+1)}\left(x_{i}\right), x_{\mathbf{f}}\right) \leq \alpha R+(1-\gamma) k_{D}\left(x_{i}, x_{\mathbf{f}}\right) .
$$

Using induction, we obtain

$$
k_{D}\left(x_{i}, x_{\mathbf{f}}\right) \leq(1-\gamma)^{i} k_{D}\left(x_{0}, x_{\mathbf{f}}\right)+\alpha R \cdot\left[\sum_{j=0}^{i-1}(1-\gamma)^{j}\right]
$$

for $i=1, \ldots, T$, and therefore we see that

$$
\begin{aligned}
k_{D}( & \left.\left(g_{\pi(T)} \circ \cdots \circ g_{\pi(1)}\right)(x), x_{\mathbf{f}}\right) \\
\quad & =k_{D}\left(x_{T}, x_{\mathbf{f}}\right)<(1-\gamma)^{T} k_{D}\left(x_{0}, x_{\mathbf{f}}\right)+\alpha R \frac{1}{\gamma} \\
\quad & (1-\gamma)^{N} d(C)+\alpha R \frac{1}{\gamma} \\
\quad & <(1-\gamma)^{N} d(C)+\frac{1}{8 K n(d(C)+1)} \cdot \frac{R_{1}}{3 R_{2}} \cdot R \cdot \frac{4 K(d(C)+1)}{R} \cdot \frac{3 R_{2}}{R_{1}} \\
\quad & (1-\gamma)^{N} d(C)+\frac{1}{2 n}<\frac{1}{2 n}+\frac{1}{2 n}=\frac{1}{n} .
\end{aligned}
$$

Hence property (P1) holds. Therefore, for $\left\{g_{t}\right\}_{t=1}^{\infty} \in \overline{\mathscr{A}_{H}^{*}}$ with

$$
d_{\mathscr{A}_{H}}\left(\left\{f_{\gamma t}\right\}_{t=1}^{\infty},\left\{g_{t}\right\}_{t=1}^{\infty}\right) \leq \alpha R,
$$

we have

$$
d_{\mathbb{A}_{H}}\left(\left\{\tilde{f}_{t}\right\}_{t=1}^{\infty},\left\{g_{t}\right\}_{t=1}^{\infty}\right)<R
$$


and $\left\{g_{t}\right\}_{t=1}^{\infty}$ satisfies (P1). This means that $\left\{g_{t}\right\}_{t=1}^{\infty} \in \mathscr{F}_{n}$ which implies, in turn, that the set $\overline{\mathscr{A}_{H}^{*}} \backslash \mathscr{F}_{n}$ is porous in $\overline{\mathscr{A}_{H}^{*}}$. The proof is complete.

\section{Convergence to a retraction}

In this section, we continue to use the notations and definitions introduced in the previous sections.

Recall that $C$ is a nonempty, closed, and convex subset of a bounded, convex domain $D$ in a Banach space $X$, and that $C$ lies strictly inside $D$. Let $F$ be a nonempty, closed, and convex subset of the set $C$. By $U_{H}^{(F)}$, we denote the set of all $f \in U_{H}$ such that $f(x)=x$ for each $x \in F$. It is obvious that $\mathcal{U}_{H}^{(F)}$ is a closed subset of $\boldsymbol{U}_{H}$. Next, we let $\mathscr{A}_{H}^{(F)}$ consist of all $\left\{f_{t}\right\}_{t=1}^{\infty} \in \mathscr{A}_{H}$ such that $f_{t} \in \mathcal{U}_{H}^{(F)}$ for all $t \geq 1$. Clearly, $\mathscr{A}_{H}^{(F)}$ is a closed subset of $\mathscr{A}_{H}$.

Assume additionally that there exists a mapping $r \in U_{H}^{(F)}$ such that $r(C)=F$.

A sequence $\left\{f_{t}\right\}_{t=1}^{\infty} \in \mathscr{A}_{H}^{(F)}$ is called normal if the following two properties hold.

(i) For each mapping $\tilde{\pi}: \mathbb{N} \rightarrow \mathbb{N}$, there exists a mapping $p_{\tilde{\pi}}: C \rightarrow F$ such that

$$
\lim _{t \rightarrow \infty}\left(f_{\tilde{\pi}(t)} \circ \cdots \circ f_{\tilde{\pi}(1)}\right)(x)=p_{\tilde{\pi}}(x)
$$

for all $x \in C$.

(ii) For each $\epsilon>0$, there exists an $N \in \mathbb{N}$ such that for each integer $T \geq N$, each mapping $\tilde{\pi}: \mathbb{N} \rightarrow \mathbb{N}$, and each $x \in C$,

$$
k_{D}\left(\left(f_{\tilde{\pi}(T)} \circ \cdots \circ f_{\tilde{\pi}(1)}\right)(x), p_{\tilde{\pi}}(x)\right) \leq \epsilon
$$

We observe that $p_{\tilde{\pi}} \in \mathcal{U}_{H}^{(F)}$ as the pointwise limit of a sequence in $u_{H}^{(F)}$ (by the compactness of $\bar{D}$ in $\sigma(X, \mathcal{N})$ and Lemma 2.6). Denote by $\mathscr{F}_{\mathcal{F}}$ the set of all normal sequences in $\mathscr{A}_{H}^{(F)}$. For $n \in \mathbb{N}$, denote by $\mathscr{F}_{n}$ the set of all $\left\{f_{t}\right\}_{t=1}^{\infty} \in \mathscr{A}_{H}^{(F)}$ for which there exists an $N \in \mathbb{N}$ such that for each $x \in C$, each integer $T \geq N$, and each mapping $\pi:\{1, \ldots, T\} \rightarrow\{1,2, \ldots\}$,

$$
\operatorname{dist}_{k_{D}}\left(\left(f_{\pi(T)} \circ \cdots \circ f_{\pi(1)}\right)(x), F\right)=\inf _{y \in F} k_{D}\left(\left(f_{\pi(T)} \circ \cdots \circ f_{\pi(1)}\right)(x), y\right)<\frac{1}{n} .
$$

It is easy to see that $\mathscr{F}=\bigcap_{n=1}^{\infty} \mathscr{F}_{n}$.

A mapping $f \in U_{H}^{(F)}$ is called normal if the constant sequence $\left\{f_{t}\right\}_{t=1}^{\infty}$, with $f_{t}=f$ for all $t \geq 1$, is normal. Denote by $\mathscr{F}^{(0)}$ the set of all normal mappings $f \in \mathcal{U}_{H}^{(F)}$.

Theorem 5.1. (i) The set $\mathscr{A}_{H}^{(F)} \backslash \mathscr{F}$ is $\sigma$-porous in $\left(\mathscr{A}_{H}^{(F)}, d_{\mathscr{A}_{H}}\right)$.

(ii) The set $U_{H}^{(F)} \backslash \mathscr{F}^{(0)}$ is $\sigma$-porous in $\left(\mathcal{U}_{H}^{(F)}, \rho \cup_{H}\right)$.

Proof. Let $n \in \mathbb{N}$ and choose $\alpha \in(0,1)$ such that

$$
\alpha<\frac{1}{4 K n(d(C)+1)} \cdot \frac{R_{1}}{3 R_{2}}
$$


(here we use notations from the previous sections). Obviously, $\alpha<1 / 4$. Choose any $\left\{f_{t}\right\}_{t=1}^{\infty}$ $\in \mathscr{A}_{H}^{(F)}$ and $R \in(0,1]$. Put

$$
\gamma=\frac{R}{2 K(d(C)+1)} \cdot \frac{R_{1}}{3 R_{2}}=s \frac{R_{1}}{3 R_{2}} .
$$

Clearly, $0<\gamma<1$ and $0<s<1$. Assume that a natural number $N>2$ is such that

$$
(1-\gamma)^{N}(d(C)+1)<\frac{1}{2 n} .
$$

Now, for each $t \in \mathbb{N}$, define

$$
f_{\gamma t}(x)=(1-s) f_{t}(x)+s r(x)
$$

for $x \in C$. Hence we have

$$
f_{\gamma t}(x)=x
$$

for every $x \in F$ and $t \geq 1$. We also have

$$
\begin{aligned}
k_{D}\left(f_{\gamma t}(y), x\right) & =k_{D}\left((1-s) f_{t}(y)+s r(y),(1-s) f_{t}(x)+s r(x)\right) \\
& \leq \max \left[k_{D}\left(f_{t}(y), f_{t}(x)\right), k_{D}(r(y), r(x))\right] \leq k_{D}(y, x)
\end{aligned}
$$

for each $x \in F, y \in C$, and $t \geq 1$. Therefore $\left\{f_{\gamma t}\right\}_{t=1}^{\infty} \in \mathscr{A}_{H}^{(F)}$ if $\left\{f_{t}\right\}_{t=1}^{\infty} \in \mathscr{A}_{H}^{(F)}$, and $f_{\gamma} \in$ $u_{H}^{(F)}$ if $f \in u_{H}^{(F)}$. Next, we get

$$
\begin{aligned}
k_{D}\left(f_{\gamma t}(x), f_{t}(x)\right) & =k_{D}\left((1-s) f_{t}(x)+s r(x), f_{t}(x)\right) \leq \frac{1}{L_{1}} s\left\|f_{t}(x)-r(x)\right\| \\
& \leq \frac{L_{2}}{L_{1}} s k_{D}\left(f_{t}(x), r(x)\right) \leq K \frac{3 R_{2}}{R_{1}} \gamma d(C) \\
& =K \frac{3 R_{2}}{R_{1}} \frac{R}{2 K(d(C)+1)} \cdot \frac{R_{1}}{3 R_{2}} d(C)<\frac{1}{2} R
\end{aligned}
$$

for $x \in C$ and $t \geq 1$, and hence for $\left\{g_{t}\right\}_{t=1}^{\infty} \in \mathscr{A}_{H}^{(F)}$ with

$$
d_{\mathscr{A}_{H}}\left(\left\{g_{t}\right\}_{t=1}^{\infty},\left\{f_{\gamma t}\right\}_{t=1}^{\infty}\right) \leq \alpha R,
$$

we obtain

$$
\begin{aligned}
d_{\mathscr{A}_{H}}\left(\left\{g_{t}\right\}_{t=1}^{\infty},\left\{f_{t}\right\}_{t=1}^{\infty}\right) & \leq d_{\mathscr{A}_{H}}\left(\left\{g_{t}\right\}_{t=1}^{\infty},\left\{f_{\gamma t}\right\}_{t=1}^{\infty}\right)+d_{\mathscr{A}_{H}}\left(\left\{f_{\gamma t}\right\}_{t=1}^{\infty},\left\{g_{t}\right\}_{t=1}^{\infty}\right) \\
& <\alpha R+\frac{1}{2} R<\frac{1}{4} R+\frac{1}{2} R<R .
\end{aligned}
$$

Let $T \geq N$ be an integer, $x \in C$, and $\pi:\{1, \ldots, T\} \rightarrow\{1,2, \ldots\}$. We will show that

$$
\operatorname{dist}_{k_{D}}\left(\left(g_{\pi(T)} \circ \cdots \circ g_{\pi(1)}\right)(x), F\right)=\inf _{y \in F} k_{D}\left(\left(g_{\pi(T)} \circ \cdots \circ g_{\pi(1)}\right)(x), y\right)<\frac{1}{n} .
$$


It is sufficient to show that

$$
\operatorname{dist}_{k_{D}}\left(\left(g_{\pi(N)} \circ \cdots \circ g_{\pi(1)}\right)(x), F\right)<\frac{1}{n} .
$$

Take $w \in C$ and $t \in \mathbb{N}$. Then, for each $z \in F$, we have $(1-s) z+s r(w) \in F$, and therefore

$$
\begin{aligned}
\operatorname{dist}_{k_{D}}\left(f_{\gamma \pi(t)}(w), F\right) & =\inf _{y \in F} k_{D}\left(f_{\gamma \pi(t)}(w), y\right) \leq k_{D}\left(f_{\gamma \pi(t)}(w),(1-s) z+s r(w)\right) \\
& =k_{D}\left((1-s) f_{\pi(t)}(w)+s r(w),(1-s) f_{\pi(t)}(z)+s r(w)\right) \\
& \leq \frac{1}{1+s R_{1} / 2 R_{2}} k_{D}(w, z)=\left(1-\frac{s R_{1}}{s R_{1}+2 R_{2}}\right) k_{D}(w, z) \\
& \leq\left(1-s \frac{R_{1}}{3 R_{2}}\right) k_{D}(w, z)=(1-\gamma) k_{D}(w, z) .
\end{aligned}
$$

This leads to

$$
\operatorname{dist}_{k_{D}}\left(f_{\gamma \pi(t)}(w), F\right) \leq(1-\gamma) \operatorname{dist}_{k_{D}}(w, F),
$$

which implies, in turn, that

$$
\begin{aligned}
\operatorname{dist}_{k_{D}}\left(g_{\pi(t)}(w), F\right) & \leq \operatorname{dist}_{k_{D}}\left(f_{\gamma \pi(t)}(w), F\right)+k_{D}\left(f_{\gamma \pi(t)}(w), g_{\pi(t)}(w)\right) \\
& \leq(1-\gamma) \operatorname{dist}_{k_{D}}(w, F)+\alpha R .
\end{aligned}
$$

By induction,

$$
\operatorname{dist}_{k_{D}}\left(\left(g_{\pi(i)} \circ \cdots \circ g_{\pi(1)}\right)(x), F\right) \leq(1-\gamma)^{i} \operatorname{dist}_{k_{D}}(w, F)+\alpha R \cdot\left[\sum_{j=0}^{i-1}(1-\gamma)^{j}\right] .
$$

Hence

$$
\begin{aligned}
\operatorname{dist}_{k_{D}} & \left(\left(g_{\pi(N)} \circ \cdots \circ g_{\pi(1)}\right)(x), F\right) \\
& <(1-\gamma)^{N} d(C)+\frac{1}{\gamma} \cdot \alpha R \\
& \leq(1-\gamma)^{N} d(C)+\frac{2 K(d(C)+1)}{R} \cdot \frac{3 R_{2}}{R_{1}} \cdot \frac{1}{4 K n(d(C)+1)} \cdot \frac{R_{1}}{3 R_{2}} \cdot R \\
& =(1-\gamma)^{N} d(C)+\frac{1}{2 n}<\frac{1}{2 n}+\frac{1}{2 n}=\frac{1}{n} .
\end{aligned}
$$

Thus we have shown that

$$
\operatorname{dist}_{k_{D}}\left(\left(g_{\pi(T)} \circ \cdots \circ g_{\pi(1)}\right)(x), F\right)<\frac{1}{n}
$$

for each point $x \in C$, each integer $T \geq N$, and each mapping $\pi:\{1, \ldots, T\} \rightarrow\{1,2, \ldots\}$. This means that $\left\{g_{t}\right\}_{t=1}^{\infty} \in \mathscr{F}_{n}$ and yields the porosity of $\mathscr{A}_{H}^{(F)} \backslash \mathscr{F}_{n}$ in $\left(\mathscr{A}_{H}^{(F)}, d_{\mathscr{A}_{H}}\right)$. Since $\mathscr{F}=\bigcap_{n=1}^{\infty} \mathscr{F}_{n}$, we conclude that $\mathscr{A}_{H}^{(F)} \backslash \mathscr{F}_{\mathscr{F}}$ is $\sigma$-porous in $\left(\mathscr{A}_{H}^{(F)}, d_{\mathscr{A}_{H}}\right)$. It is not difficult to see that the second statement of the theorem has also been proved. 
Remark 5.2. Finally, we observe that all the results proved in this paper have a metric character and therefore analogous results are valid in appropriately defined spaces of sequences of $k_{D}$-nonexpansive (not necessarily holomorphic) mappings.

\section{Acknowledgments}

The work of the second author was partially supported by the Israel Science Foundation founded by the Israel Academy of Sciences and Humanities (Grant 592/00), by the Fund for the Promotion of Research at Technion, and by the Technion VPR Fund. Both authors are very grateful to Tadeusz Kuczumow for many helpful and fruitful discussions.

\section{References}

[1] Y. Benyamini and J. Lindenstrauss, Geometric Nonlinear Functional Analysis. Vol. 1, American Mathematical Society Colloquium Publications, vol. 48, American Mathematical Society, Rhode Island, 2000.

[2] M. Budzyńska, T. Kuczumow, and T. Sękowski, Total sets and semicontinuity of the Kobayashi distance, Nonlinear Anal. 47 (2001), no. 4, 2793-2803, Proceedings of the Third World Congress of Nonlinear Analysts, Part 4 (Catania, 2000).

[3] S. B. Chae, Holomorphy and Calculus in Normed Spaces, Monographs and Textbooks in Pure and Applied Mathematics, vol. 92, Marcel Dekker, New York, 1985.

[4] F. S. De Blasi and J. Myjak, Sur la porosité de l'ensemble des contractions sans point fixe [On the porosity of the set of contractions without fixed points], C. R. Acad. Sci. Paris Sér. I Math. 308 (1989), no. 2, 51-54 (French).

[5] _ On a generalized best approximation problem, J. Approx. Theory 94 (1998), no. 1, 5472.

[6] F. S. De Blasi, J. Myjak, and P. L. Papini, Porous sets in best approximation theory, J. London Math. Soc. (2) 44 (1991), no. 1, 135-142.

[7] R. Deville and J. P. Revalski, Porosity of ill-posed problems, Proc. Amer. Math. Soc. 128 (2000), no. $4,1117-1124$.

[8] N. Dunford, Uniformity in linear spaces, Trans. Amer. Math. Soc. 44 (1938), no. 2, 305-356.

[9] C. J. Earle and R. S. Hamilton, A fixed point theorem for holomorphic mappings, Global Analysis (Proc. Sympos. Pure Math., Vol. XVI, Berkeley, Calif, 1968), American Mathematical Society, Rhode Island, 1970, pp. 61-65.

[10] T. Franzoni and E. Vesentini, Holomorphic Maps and Invariant Distances, Notas de Matemática, vol. 69, North-Holland, New York, 1980.

[11] K. Goebel and S. Reich, Uniform Convexity, Hyperbolic Geometry, and Nonexpansive Mappings, Monographs and Textbooks in Pure and Applied Mathematics, vol. 83, Marcel Dekker, New York, 1984.

[12] L. A. Harris, Schwarz-Pick systems of pseudometrics for domains in normed linear spaces, Advances in Holomorphy (Proc. Sem. Univ. Fed. Rio de Janeiro, Rio de Janeiro, 1977), NorthHolland Math. Stud., vol. 34, North-Holland, New York, 1979, pp. 345-406.

[13] M. Jarnicki and P. Pflug, Invariant Distances and Metrics in Complex Analysis, de Gruyter Expositions in Mathematics, vol. 9, Walter de Gruyter, Berlin, 1993.

[14] J. Kapeluszny and T. Kuczumow, A few properties of the Kobayashi distance and their applications, Topol. Methods Nonlinear Anal. 15 (2000), no. 1, 169-177.

[15] S. Kobayashi, Invariant distances on complex manifolds and holomorphic mappings, J. Math. Soc. Japan 19 (1967), 460-480.

[16] T. Kuczumow, The weak lower semicontinuity of the Kobayashi distance and its applications, Math. Z. 236 (2001), no. 1, 1-9. 
[17] T. Kuczumow, S. Reich, and D. Shoikhet, Fixed points of holomorphic mappings: a metric approach, Handbook of Metric Fixed Point Theory (W. A. Kirk and B. Sims, eds.), Kluwer Academic, Dordrecht, 2001, pp. 437-515.

[18] T. Kuczumow and A. Stachura, Iterates of holomorphic and $k_{D}$-nonexpansive mappings in convex domains in $\mathbf{C}^{\mathrm{n}}$, Adv. Math. 81 (1990), no. 1, 90-98.

[19] S. Reich and A. J. Zaslavski, Convergence of generic infinite products of nonexpansive and uniformly continuous operators, Nonlinear Anal. Ser. A: Theory Methods 36 (1999), no. 8, 10491065.

[20] - The set of divergent infinite products in a Banach space is $\sigma$-porous, Z. Anal. Anwendungen 21 (2002), no. 4, 865-878.

[21] L. Zajíček, Porosity and $\sigma$-porosity, Real Anal. Exchange 13 (1987/1988), no. 2, 314-350.

[22] - Products of non- $\sigma$-porous sets and Foran systems, Atti Sem. Mat. Fis. Univ. Modena 44 (1996), no. 2, 497-505.

[23] Small non- $\sigma$-porous sets in topologically complete metric spaces, Colloq. Math. 77 (1998), no. 2, 293-304.

Monika Budzyńska: Instytut Matematyki, Uniwersytet Marii Curie-Skłodowskiej (UMCS), 20-031 Lublin, Poland

E-mail address: monikab@golem.umcs.lublin.pl

Simeon Reich: Department of Mathematics, Technion - Israel Institute of Technology, 32000 Haifa, Israel

E-mail address: sreich@techunix.technion.ac.il 


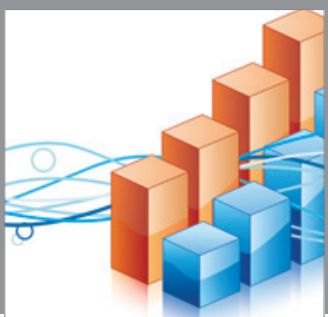

Advances in

Operations Research

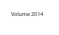

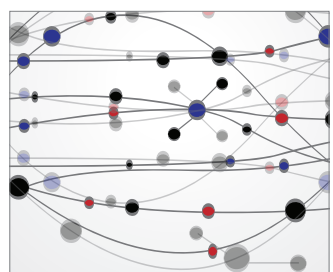

\section{The Scientific} World Journal
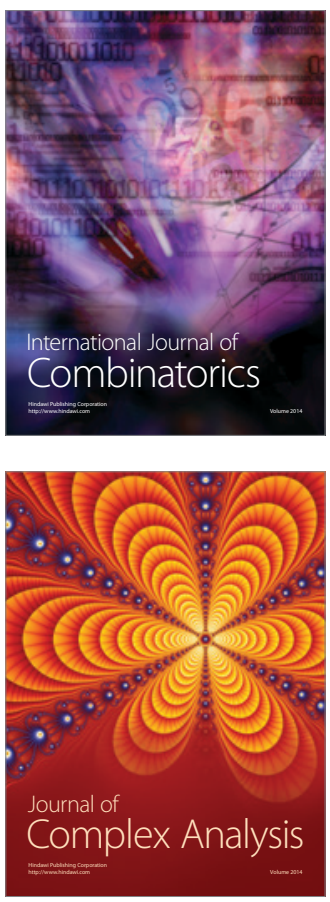

International Journal of

Mathematics and

Mathematical

Sciences
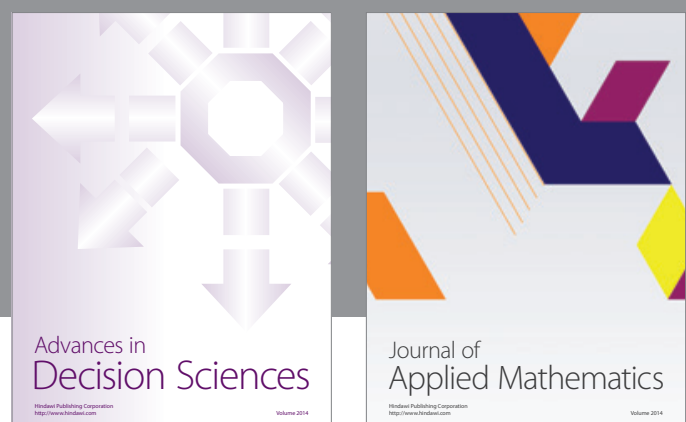

Journal of

Applied Mathematics
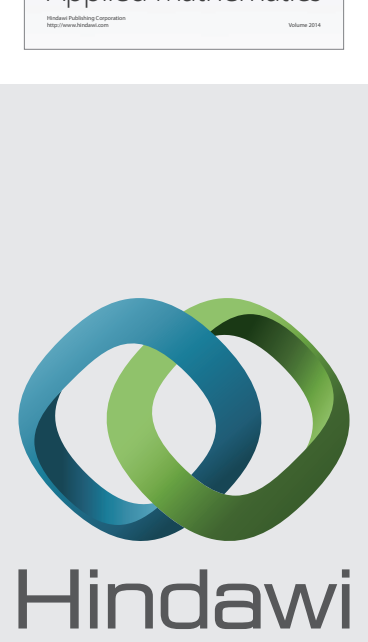

Submit your manuscripts at http://www.hindawi.com
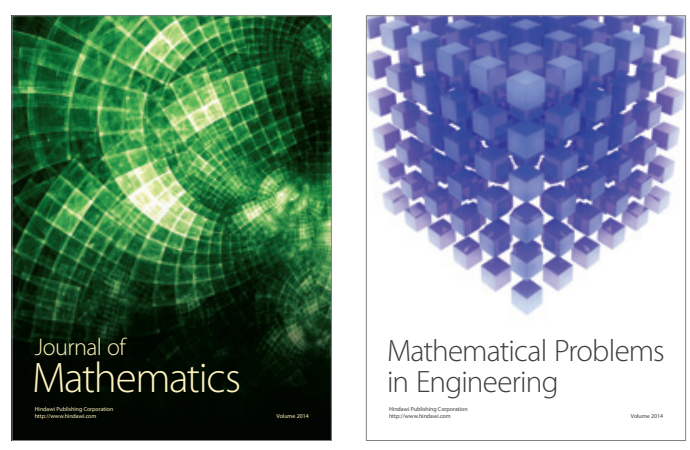

Mathematical Problems in Engineering
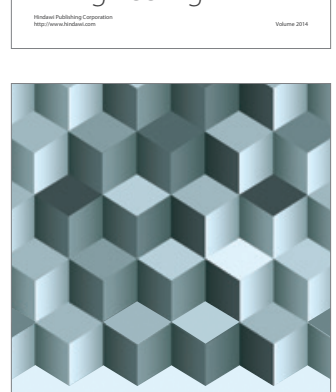

Journal of

Function Spaces
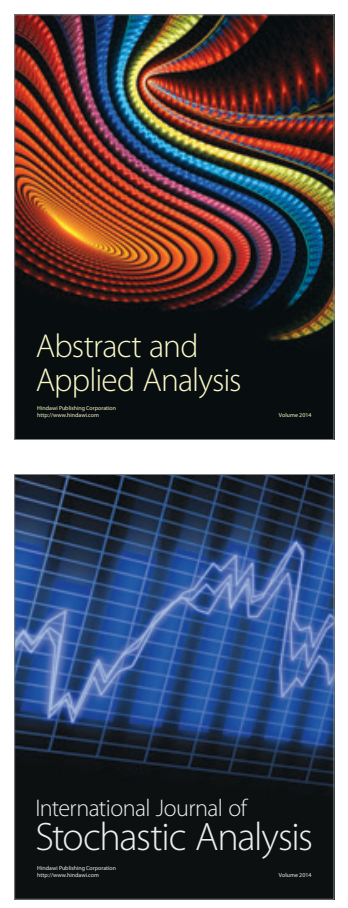

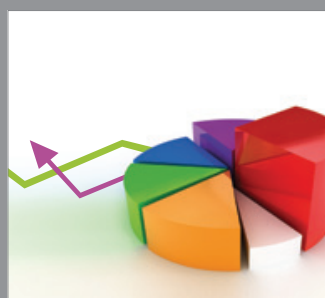

ournal of

Probability and Statistics

Promensencen
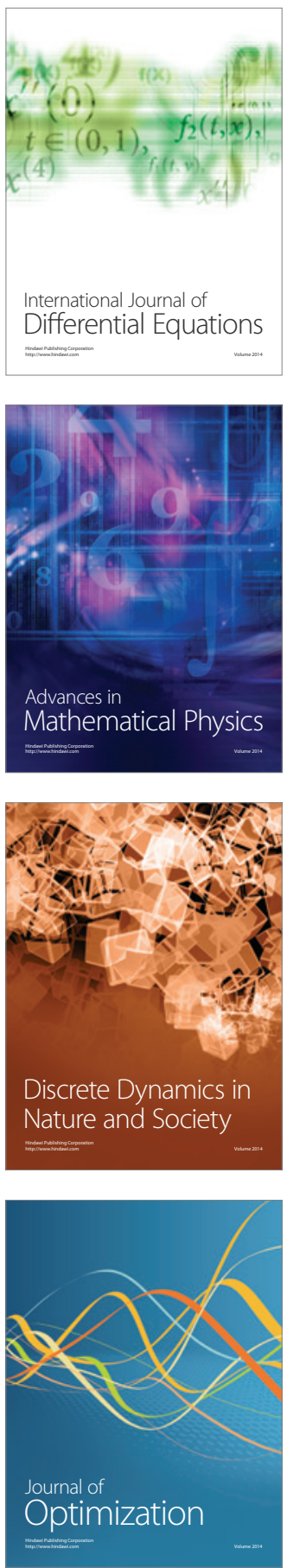